\title{
Development of TanDEM-X DEM Calibration Concept
}

\author{
J. Hueso González ${ }^{1}$, M. Bachmann ${ }^{1}$, H. Fiedler ${ }^{1}$, S. Huber ${ }^{1}$, G. Krieger ${ }^{1}$, B. Wessel ${ }^{2}$, M. Zink ${ }^{1}$ \\ German Aerospace Center (DLR) \\ ${ }^{1}$ Microwaves and Radar Institute - Oberpfaffenhofen \\ ${ }^{1}$ German Remote Sensing Data Center - Oberpfaffenhofen \\ 82234 Weßling - Germany \\ Fax: +498153281449 \\ jaime.hueso@dlr.de
}

\begin{abstract}
The TanDEM-X mission [1] comprises two fully active synthetic aperture radar satellites operating in $X$-band. The primary goal of this mission is the derivation of a high-precision global Digital Elevation Model (DEM) according to HRTI level 3 quality [2]. This requires accurate calibration of the interferometric system parameters, where the interferometric height is determined by the phase difference between the two images and the spatial geometry. Content of this paper is the development of a general concept for this calibration, which has a key incidence on mission aspects like the data acquisition plan and the data take adjustment and mosaicing.
\end{abstract}

\section{INTRODUCTION AND OBJECTIVES}

The challenge of calibrating the TanDEM-X DEM lies on the complexity of the system and the strong height accuracy requirements. The interferometric height, which the DEM is based on, depends on the phase difference between the two bistatic images and on the spatial geometry. Additionally, baseline errors intrinsic of the bi-static SAR configuration combined with errors and drifts of the radar instrument introduce errors in the interferometric height. Thus, the DEM has to be corrected to achieve the accuracies defined (Table 1). The correction techniques take into consideration the nature of the errors and allow correctly adjusting and combining the data takes that cover a certain Earth region and building an accurate height model.

TABLE I

TANDEM-X DEM SPECIFICATIONS

\begin{tabular}{|l|l|l|}
\hline Requirement & Specification & HRTI-3 \\
\hline $\begin{array}{l}\text { Absolute vertical } \\
\text { accuracy (global) }\end{array}$ & $90 \%$ linear error & $10 \mathrm{~m}$ \\
\hline $\begin{array}{l}\text { Relative vertical } \\
\text { accuracy } \\
(100 \mathrm{~km} \times 100 \mathrm{~km})\end{array}$ & $\begin{array}{l}90 \% \text { linear point- } \\
\text { to-point error }\end{array}$ & $2 \mathrm{~m}($ slope $<20 \%)$ \\
$4 \mathrm{~m}($ slope $>20 \%)$
\end{tabular}

The final potential of the calibration concept developed for the TanDEM-X Preliminary Design Review is to extend the strategy for the data acquisition plan [3] in order to obtain global coverage within the mission time and to provide in parallel sufficient additional information to allow the application of bundle block corrections [4] to the raw DEMs.

\section{ERROR SOURCES}

The main sources of the arising phase errors mentioned before can be classified into three groups: inaccuracies in the baseline determination, phase errors in the radar instruments and performance degradation due to both noise equivalent $\sigma_{0}$ (NESZ) and signal to noise ratio (SNR). Noise-like, randomly distributed contributions of these errors already exhaust most of the $2 \mathrm{~m}$ relative error margin allowed for an area of $100 \mathrm{~km} \times 100 \mathrm{~km}$. In order to keep the total error under this requirement, a relative error of $0.5 \mathrm{~m}$ has been defined as the threshold for the "low frequency" height errors.

Hence, to achieve the required accuracy, "low frequency" parts of the errors which appear as biases or drifts in terms of the length of one data take have to be minimised. These "low frequency" error parts mainly come from two sources, the baseline determination and the instrument phase drifts.

The slow changing baseline errors for baseline errors are inaccuracies in the precise determination of the relative orbit, which is the three dimensional distance between both satellites orbiting in the TanDEM helix formation. The required accuracy for this calculation is of less than a millimeter. The attitude determination is a second critical point although it mainly generates an offset as well as variations in the SAR antenna phase centre.

On the instrument side, slow errors occur due to remaining interpolation errors after internal calibration. The internal calibration is used to correct systematic drifts within the instruments, exemplary caused by temperature drifts of the amplifiers in the transmit/receive modules. Also in the synchronisation path, residual drift errors occur. The synchronisation loop is used to measure relative drifts in the satellites oscillators. As the loop is not covered by the internal calibration correction, temperature drifts in the amplifiers have to be corrected using house keeping data of temperature sensors on these amplifiers, which only have a finite accuracy. 
When a data take is acquired, these phase errors lead to a height error in the resulting raw DEM. For the further DEM processing, it is important to know how these errors develop within the raw DEMs. Knowing the impact of these errors on the shape, an adjustment can be performed to further minimise the errors, taking neighbouring strips into account as well as crossing orbits.

\section{CALIBRATION APPROACH}

The mission scenario for TanDEM-X is designed to obtain a global coverage of the earth within mission time, and to guarantee a DEM with the specified accuracies. Hence, the designed mission plan foresees that all land surfaces will be covered at least twice with different heights of ambiguity $\left(\mathrm{h}_{\mathrm{amb}}\right)$ to support multi-baseline phase unwrapping (PU). The northern hemisphere will be mapped with ascending orbits, whereas the southern hemisphere with descending orbits. The length of the data takes will be maximized within the resource limits in order to simplify the adjustment.

All these fundaments give a good overview of the data take scenario the DEM calibration will face.

The phase errors introduced in chapter 2 would imply height errors in the raw DEM that trespass the specified height error threshold of the mission. Therefore, several data take adjustments and calibration strategies had to be considered.

Relative corrections can be derived from concurring swath overlaps and crossing orbits in the data take scenario, by means of a bundle block adjustment. Especially developed bundle block mosaicing techniques are applied in different ways depending on the scenario configuration to balance the height error realisations.

Absolute height calibration requires accurate height references. The references have to be adequately distributed depending on the data take adjustment scenario.

Coverage on all significant isolated land masses and a controlled accuracy are pursued, with the aim of guaranteeing the correct adjustment of the elevation models by the Mosaicing and Calibration Processor (MCP).

This can be achieved by using global data sets, which provide very useful information even in regions of the planet where the access to height data is limited or unreliable.

However, local height calibration targets are still necessary, due to their high accuracy, particularly in regions where global data may have blind spots and for validation purposes. As a secondary mission goal of TanDEM-X, in certain interest regions the DEM accuracy specifications will be improved to fulfil a HRTI-4 standard. In these cases, local very accurate calibration targets may play an important role.

Reference information in open terrain is preferable, because uncertainties between terrain and surface models do not need to be considered.

Several absolute calibration references have been studied:
- GPS Tracks have already been used during SRTM acquisition. These provide an accuracy of several centimetres but are not available globally or have to be acquired externally for this purpose.

- Space-borne Altimeter data as measured by the ICESat Global Laser Altimeter Scanner provide accurate, globally distributed height information as well as evaluation and classification information for each measurement point.

- Local, high resolution DEMs were available and accessible. The drawback is the high costs of these DEMs.

- Ground Calibration Targets like corner reflectors, which are especially suitable for being placed on difficult or mountainous terrain.

- An airport data base providing the absolute heights of 40.000 airports worldwide.

A combination of these absolute references will provide a good coverage for hooking in the DEM.

\section{ERROR SimUlation AND ADJUSTMENT TOOLS}

A height error simulation tool has been developed taking into account the above mentioned inputs. With its help it is possible to analyse the influence of the different error sources on the system performance and to propose inputs to the acquisition scenario. The simulator includes an error generator and an adjustment module, which applies the bundle block techniques selected by the user to the calculated height error realisation. They will give an idea of the number, quality and distribution of calibration references and of crossing orbits required to successfully calibrate the global DEM.

First, a scenario containing only parallel orbits will be adjusted. As described in chapter 2, the acquisition plan proposes to obtain two acquisitions for each orbit, which would offer an adjusting scenario similar to the one presented in Figure 1.

The parallel scenario has three major advantages:

- The same strips are mapped during the same period of time in the year. Hence, vegetation, tree cover or ice cover are seen in a similar state.

- No major formation change will be necessary during the first two years of acquisition. First after these two years, the satellite formation is changed anyway to acquire difficult terrain like mountainous regions.

- Multi baseline processing is simplified significantly.

At least four ground control points should be situated within the scenario. The ground control points can be distributed within one data take or at the borders of the data takes in the scene, so they can be completely adjusted. The central data takes will be corrected by propagating the information of the external ones fixed by control points. All the control points have an accuracy of $50 \mathrm{~cm}$ (one sigma). 


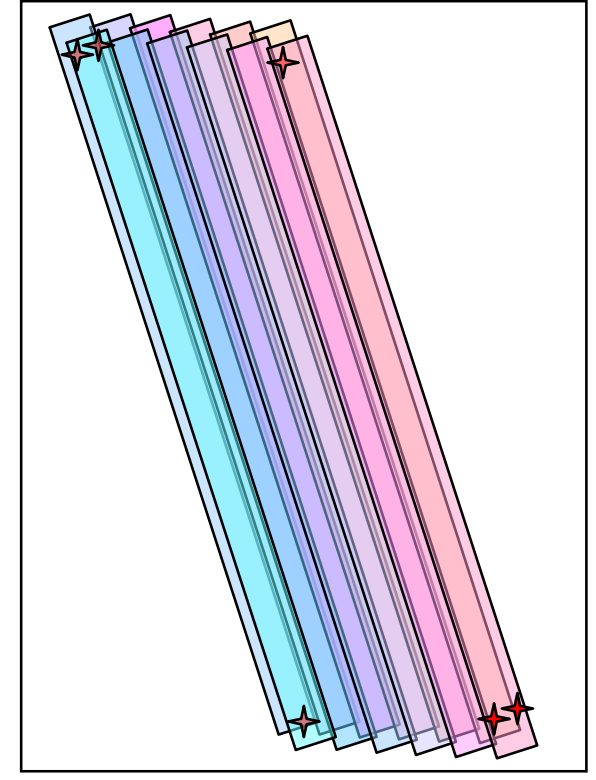

Figure 1: Double adjacent orbits.

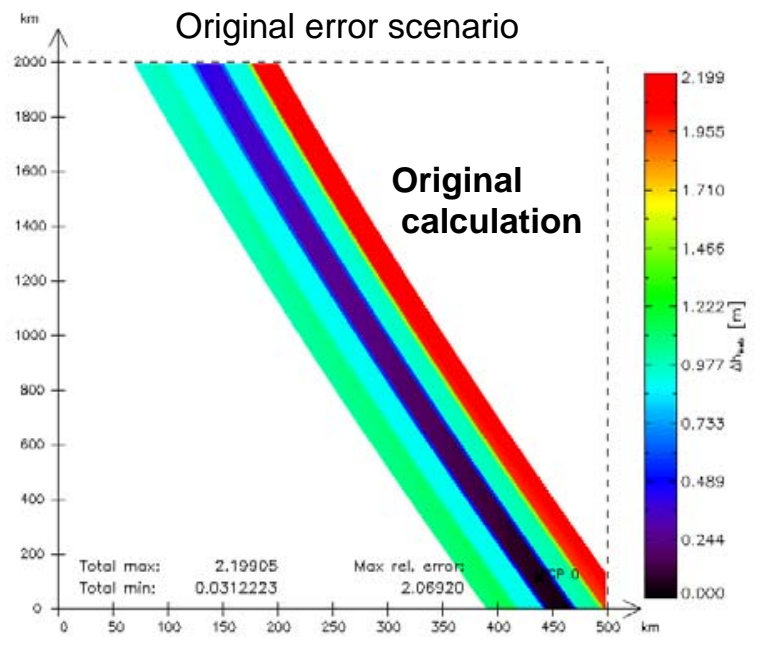

Figure 2.a: Error realisation and adjustment.

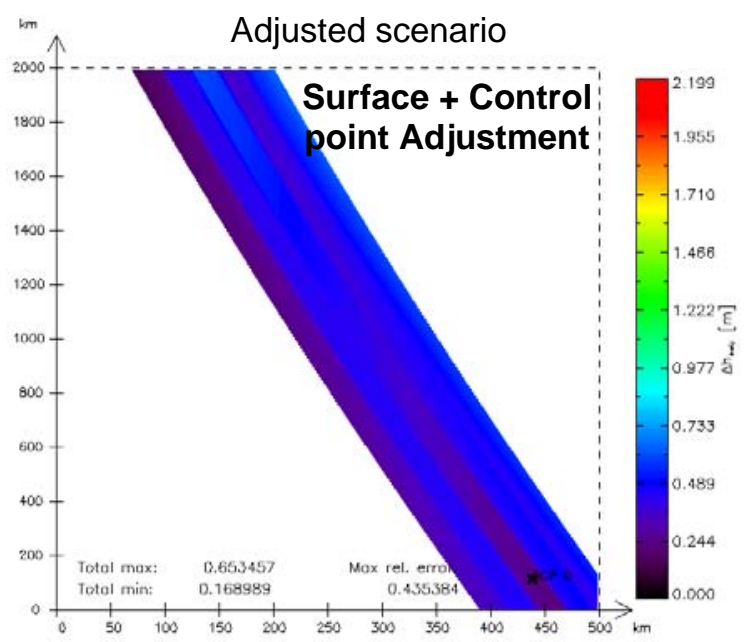

Figure 2.b: Error realisation and adjustment.
One calculated error realisation is shown in Figure 2.a, together with the results of the adjustment (2.b). The maximum relative error has been improved from $2.0 \mathrm{~m}$ to $0.43 \mathrm{~m}$, which is a very good correction and keeps the height error within the requirements.

If there is no appropriate amount of ground control points accessible in one region, crossing orbits should be positioned within the length of the adjacent orbits. The advantage of crossing orbits is that they allow correcting the elevation tilts of the other realisations with less ground control points as in the last example and more flexibility in their location.

Two ground control points are needed in the crossing orbits, one on each extreme, as depicted in Figure 3. This compensates the along track drift of the crossing orbit, which will be the reference for the others. Another important conclusion of this simulation is the required distance between the crossing orbits in order to get a good error correction.

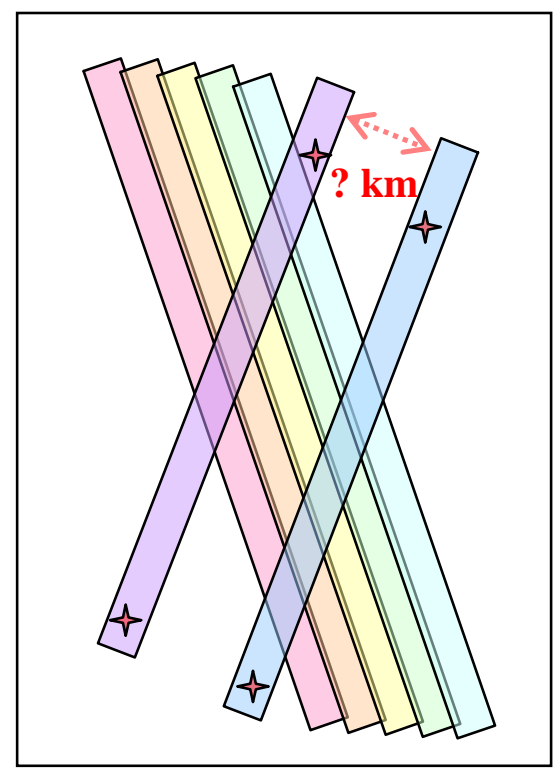

Figure 3: Ascending orbits scene with two crossing orbits

Figure 4 shows the simulation results. The maximum relative error has improved from $2 \mathrm{~m}$ to $0.38 \mathrm{~m}$, which meets our requirements of $0.5 \mathrm{~m}$. Some refinements in the adjustment scene are still possible. A better correction of the tilt could be achieved with an extra ground control point in the crossing data takes. The residual along track slope could be levelled by separating a little more the crossing data takes.

The separation between crossing orbits depends on the size of the adjacent scenario to adjust and on the angle between ascending and descending orbits, which varies with the latitude of the acquisition. Assuming the simulation parameters, a good separation between crossing orbits lies at a distance of about $1000 \mathrm{~km}$. The position of the crossing orbits should be chosen such, that, at least by these data takes some ground control points are covered. 


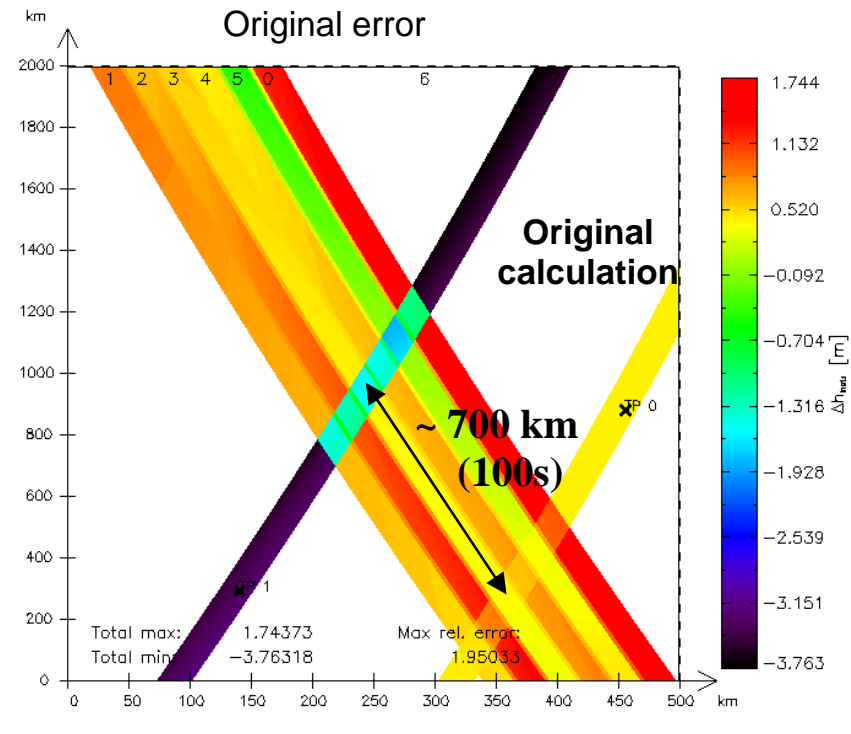

Figure 4.a: Error realisation and adjustment.

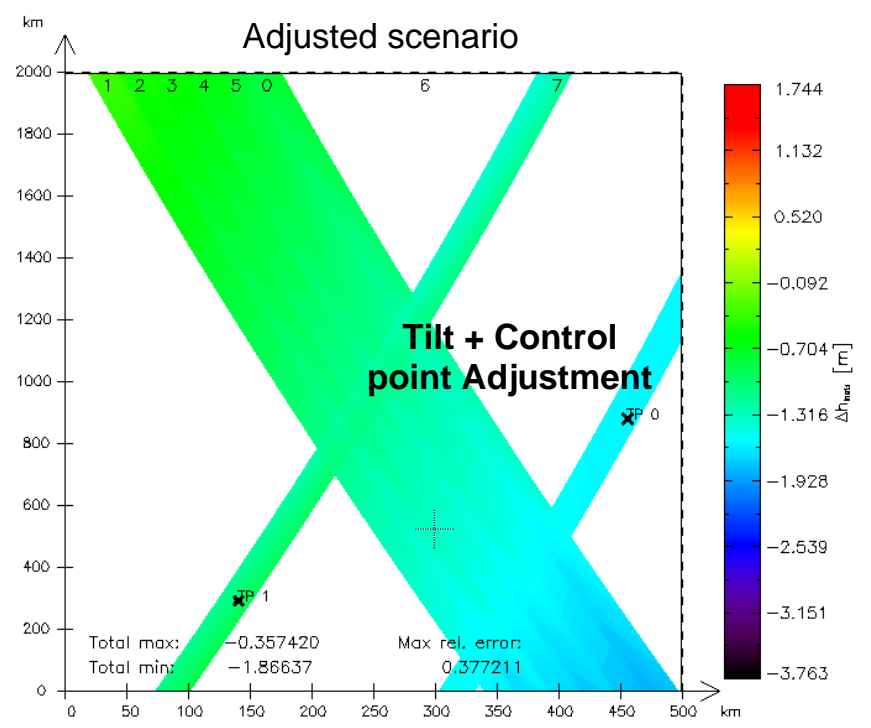

Figure 4.b: Error realisation and adjustment.
Constraints for these simulations are that no high frequent noise was considered. Also, the simulations have to be repeated with a statistically higher number of individual error realisations to get a clear view of statistical impact on the errors.

\section{SUMMARY AND CONCLUSIONS}

As a preliminary step, all the phase errors caused by baseline uncertainties and instrument drifts have been carefully analysed and implemented in a new software tool.

Some "key" scenarios have been simulated and adjusted in order to validate the bundle block adjustment techniques and assess the viability of the mission scenarios and the achievement of the desired height accuracies. This has been useful to provide important inputs to the mission and acquisition plan.

During 2007, after the launch of the first satellite of the tandem, TerraSAR-X, its calibration and validation results will offer essential information about the satellite operation, which will allow refining the performance prediction of the whole TanDEM-X system. These data will also be used to validate and improve the calibration concept presented in this paper.

\section{ACKNOWLEDGMENT}

The TanDEM-X project is partly funded by the German Federal Ministry for Economics and Technology (Förderkennzeichen 50 EE 0601).

\section{REFERENCES}

[1] A. Moreira et al. "TanDEM-X: A TerraSAR-X Add-On Satellite for Single-Pass SAR Interferometry”, in Proc. IGARSS, Anchorage, USA, 2004.

[2] “HRTI-3-DEM draft document”, NGA, MIL PRF 89048.

[3] H. Fiedler, G. Krieger, M. Werner, K. Reiniger, M. Eineder, S. D'Amico, R. Klein, D. Erhardt, M. Wickler. "The TanDEM-X Mission Design and Data Acquisition Plan”, EUSAR, Dresden, Germany, 2006.

[4] B. Triggs, P. McLauchlan, R. Hartley, and A. Fitzgibbon. "Bundle adjustment - a modern synthesis. Vision Algorithms: Theory \& Practice”. Springer-Verlag, 2000. 